\title{
Searching for Millisecond Pulsar Binaries Among Fermi Sources
}

\section{Z. Wang*, X. Dai, and Y. Xing}

Shanghai Astronomical Observatory, Chinese Academy of Sciences, 80 Nandan Road, Shanghai 200030, China

E-mail: wangzxeshao.ac.cn

The Fermi Gamma-Ray Space Telescope (Fermi) has opened a new window for our studies of pulsars, particularly the millisecond pulsars (MSPs). Among the sources detected by Fermi, a significant number of pulsars should exist and have not been identified. We have carried out a candidate MSP selection based on the known properties of pulsars, and Fermi data analysis for the selected sources for further selection has also been conducted. We propose to search for the MSP binaries among them through optical observations by finding their orbital flux modulation. The proposed optical observations would be an alternatively effective method for finding MSP binaries, particularly if they are eclipsing systems. The observations could be a good program carried out with the Southern African Large Telescope (SALT) given SALT's capabilities.

SALT Science Conference 2015 -SSC2015-

1-5 June, 2015

Stellenbosch Institute of Advanced Study, South Africa

\footnotetext{
*Speaker.
} 


\section{Introduction}

Launched in 2008 June, the Fermi Gamma-Ray Space Telescope (Fermi) has allowed us to study the $\gamma$-ray sky with much improved spatial resolution and sensitivity, comparing to former Gamma-ray telescopes. The main instrument onboard Fermi is the Large Area Telescope (LAT; [5]), which works in the energy range from $100 \mathrm{MeV}$ to $300 \mathrm{GeV}$ and has been scanning the whole sky every three hours. Its observations have thus far resulted in a third catalog that contains 3033 sources [2]. While most of the identified or associated are Active Galactic Nuclei (AGN; [3]), the prominent class in the Milky Way is pulsars.

In the second Fermi LAT catalog of $\gamma$-ray pulsars [1], 117 pulsars are formally reported to have $\gamma$-ray pulsations detected. The results have confirmed the long expectation that pulsars are the main $\gamma$-ray sources in the Milky Way, both on the basis of both observational results from previous Gamma-Ray telescopes (e.g., [24]) and theoretical studies (e.g., [9, 12]). In addition to establishing the $\gamma$-ray pulsar population, Fermi has particularly improved our studies of millisecond pulsar (MSP) binaries by finding candidates. For example, in Figure 1 that shows all MSP binaries, there were only two eclipsing MSP binaries for a long time. They were PSR B1957+20 [10] and PSR J2051-0827 [20]. These two are also called "black widow" systems because the material that eclipses the pulsation signals over orbital phases should come from the companion star, which implies that the companion is under ablation by the pulsar wind of the central MSP. Now with Fermi's help, the number of such eclipsing binaries has been increased by more than six times (e.g., [17]), which includes a sub-class of so-called "redbacks" [17].

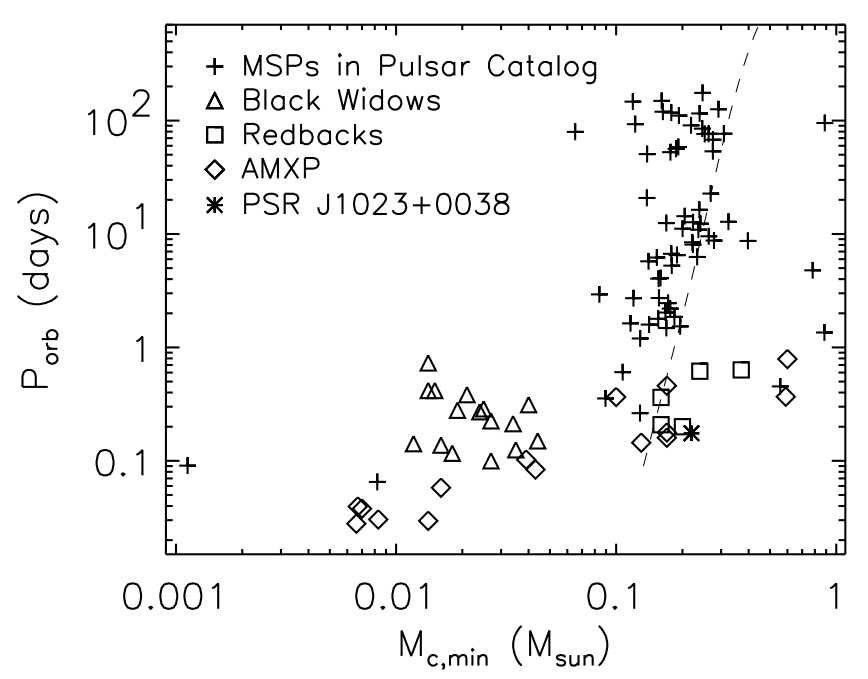

Figure 1: Known MSP binaries, where $M_{c, \min }$ is the minimum mass of a companion and $P_{\text {orb }}$ is the orbital period in units of days. For completeness, the known accreting millisecond X-ray pulsars (AMXPs; [13]) are also included.

Among 3033 LAT sources, 992 have not been identified or found to be associated with any known objects that are likely $\gamma$-ray sources [2]. A significant number of pulsars must be among them. For example, based on the current Fermi LAT source identification, 100 LAT sources with low Galactic latitudes should be young pulsars. For the high Galactic sources, MSPs are likely 
hidden among them because MSPs are isotropically distributed in the sky. However it takes effort to identify particularly the eclipsing MSPs since the intrabinary material can severely weaken the pulsation signals at radio frequencies (see, e.g., [15]). Here we propose to use optical observations for finding new MSP binaries, a method which has been more or less successfully used (e.g., [18, 22]). In Section 2, we describe our selection of candidate MSP sources from the Fermi LAT source catalog and provide the preliminary results from our analysis of the LAT data of the candidates. In Section 3, we present our estimates for possible optical modulation from black widows and redbacks, considering that they exist among the candidates. We discuss the possible use of the Southern African Large Telescope (SALT) for the proposed observations in Section 4.

\section{Target Selection and Data Analysis}

On the basis of Fermi LAT results, pulsars are known to be different from AGN at $\gamma$-ray energies with two general properties. First, pulsars have stable emission, not showing the strong variability AGN have (e.g., [27]). Secondly, a pulsar's emission can be described by an exponentially cutoff power law with the cutoff energies at several GeV [1], while AGN generally have 'straight' power law spectra (e.g., [3]). The differences are well illustrated in Figure 2.

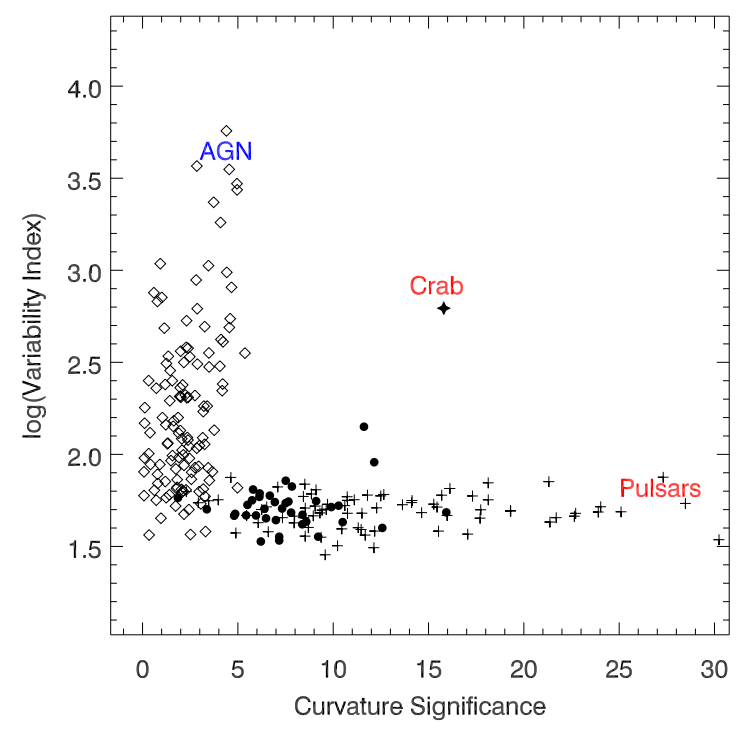

Figure 2: Illustration of properties of spectral curvature and flux variability for AGN (diamonds) and pulsars (plus signs). Bright LAT sources are shown here, which include a few unidentified sources (black dots). Because of the property differences, AGN and pulsars are well separated in the figure.

Using these properties, we have selected candidate MSP systems from the third LAT catalog. The criteria are 1) the Galactic latitudes are greater than 5 degrees, which helps exclude young pulsars; 2 ) the variability indices are $\leq 72.44$ (99\% confidence for a source not being a variable; [2]); 3) the curvature significances are $>3 \sigma$ [2]. The latter two help exclude AGN. The selection results in 101 sources from the LAT catalog, whose Galactic positions are shown in Figure 3. As can be seen, the sources are concentrated towards the Galactic center direction, as nearly $40 \%$ of 
them are located within Galactic longitudes of \pm 30 degrees. This anisotropic distribution suggests that they are likely associated with the Milky Way, and our selection works possibly properly.

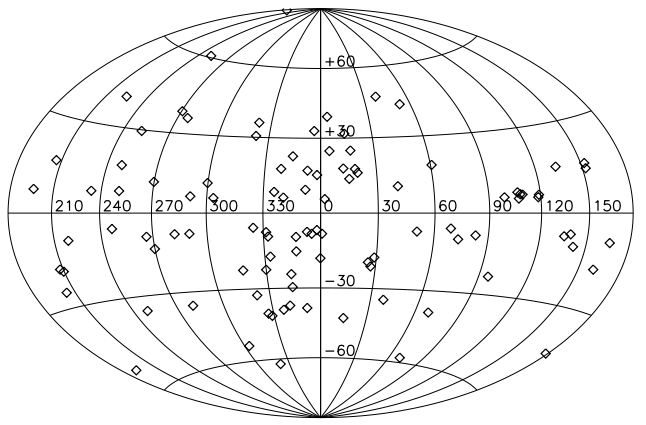

Figure 3: Galactic positions of the selected 101 sources from the third LAT catalog. Nearly $40 \%$ of them are located within Galactic longitudes of \pm 30 degrees.

The LAT catalog resulted from complicated data analysis processes described in [2], and further analysis confirmation is needed for the 101 selected sources. We have downloaded and used $6.5 \mathrm{yr}$ Fermi LAT data, and carried out the detailed analysis for each of them. Thus far 39 of them have been studied. The details will be presented elsewhere by Dai et al. (2015, in preparation). In a summary, based on the Test Statistic (TS) maps and spectral analysis, 16 sources have been found to be nice point sources and have pulsar-type spectra (i.e., well fit with an exponentially cutoff power law). Furthermore they did not show significant flux variations over the $6.5 \mathrm{yr}$ time period. These sources are thus good candidate MSPs.

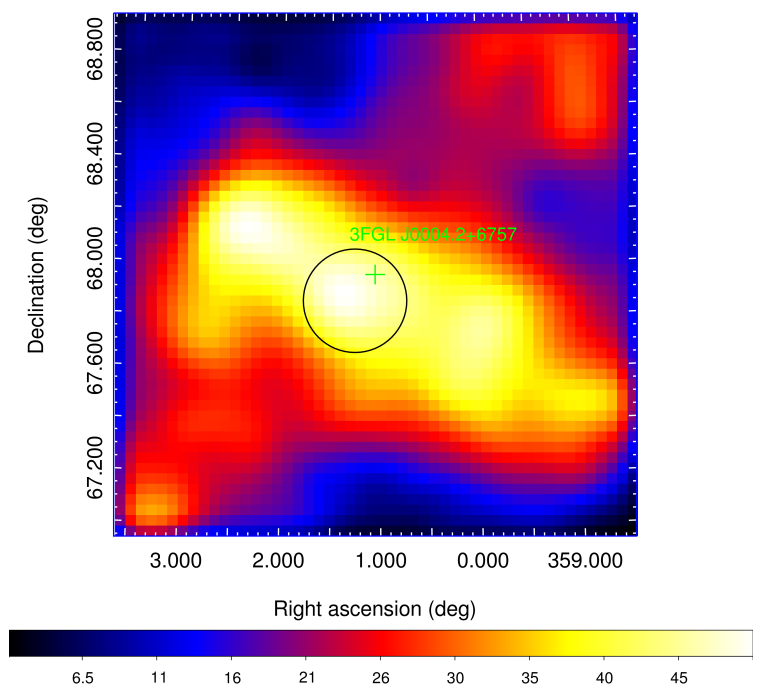

Figure 4: Example of a selected source that is likely contaminated by two nearby objects, which makes the catalog results not correct. The green plus marks the position of the source given in the LAT catalog. Such sources are excluded by our data analysis. 


\section{MSP Modulation Estimates}

One class of interesting MSP binaries are black widows and redbacks. Their presence, including the transitional properties for two redbacks PSR J1023+0038 [4] and XSS J12270-4859 [6], challenges our understanding for the formation process of MSPs (e.g., [8, 7]). It is warranted to find more of them among Fermi sources, particularly given that PSR J1023+0038 and XSS J12270-4859 have detectable $\gamma$-ray emission and showed related flux changes when they had state transitions $[23,19,11,28]$.

It is extremely difficult to find millisecond pulsation signals in Fermi LAT data via blind searches [14]. On the other hand, while radio searches are the main tool for confirming $\gamma$-ray MSPs [1], pulsation signals can be severely weakened because of intrabinary material in the eclipsing MSP binaries (e.g., $[15,22]$ ). In such a binary, since the companion is tidally locked such that rotation is synchronous with the orbit, the inner surface of the companion is strongly irradiated by the pulsar wind from the MSP. As a result, the visible area of the heated surface varies as a function of orbital phase and the binary would show sinusoidal-like optical flux modulation (e.g., $[21,16,25])$. Therefore we may search for them alternatively through optical observations by finding optical variable sources in the fields of targets (e.g., [18, 22]).

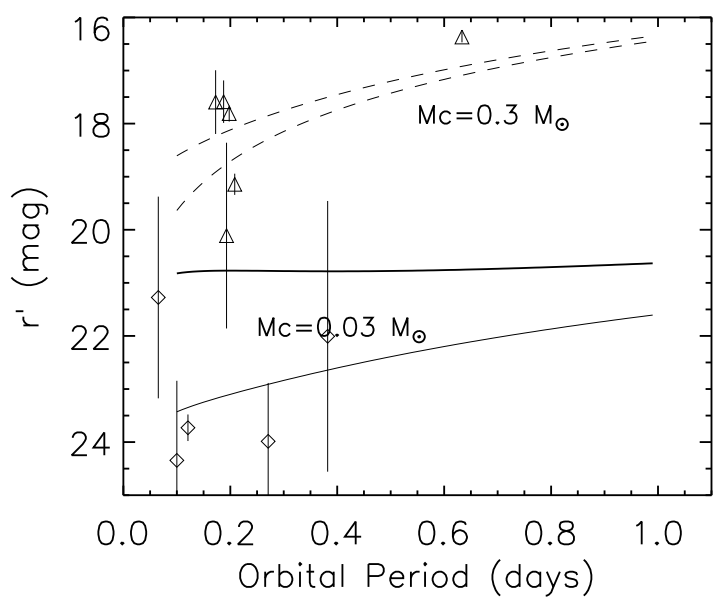

Figure 5: Estimated optical $r^{\prime}$ band brightnesses and variations as a function of orbital periods for black widows (the two solid curves mark the variation range) and for redbacks (the two dashed curves mark the variation range). 11 black widows (diamonds) and redbacks (triangles) with their representative magnitude ranges (scaled to $1.5 \mathrm{kpc}$ ) are overplotted, indicating that our estimation approximately describes the possible brightness ranges for these two classes of MSP binaries.

The difference between black widows and redbacks is their companion mass. While the former generally consist of a $\sim 0.03 M_{\odot}$ companion star, the latter have relatively massive, $\sim 0.3 M_{\odot}$ companions (see Figure 1). The current known black widows and redbacks all have orbital periods in a range of 0.1-1.0 days. Assuming canonical $1.4 M_{\odot}$ for the masses of neutron stars in such binaries, the binary separation distances and Roche-lobe radii of the companions are simply a function of the orbital periods. For our selected targets, we may assume that the source distance is $\sim 1.5 \mathrm{kpc}$ (middle value for known $\gamma$-ray MSPs; see [1]; note that the spin-down energies $\dot{E}$ of MSPs are generally low and the detectable MSPs cannot be far away). The radius of a companion 
is considered to be $85 \%$ of the Roche-lobe radius ([21, 16]; actually for the redback J1023+0038, the companion overfills its Roche lobe). Then assuming $\dot{E} \simeq 10^{34} \mathrm{erg} \mathrm{s}^{-1}$ and a value of 0.5 for the companion's albedo, the effective temperature of the heated side of the companion can be obtained (see, e.g., [26], for details), where the base temperature of $3000 \mathrm{~K}$ or $5000 \mathrm{~K}$ (the non-irradiated side) are assumed for the companion in a black widow [21, 16] or a redback [25], respectively. The resulting $r^{\prime}$ magnitudes, as a function of orbital periods, are shown in Figure 5. For black widow binaries, they would be in a range of $r^{\prime} \simeq 20.5-23.5$, with variations in a range of 1-2.5 mag, while for redback binaries, they would have $r^{\prime} \simeq 16.5-20$, with variations of $0.1-1.0$ mag. These variations are detectable with different observing strategies applied depending on the size of a telescope.

\section{Discussion}

We therefore propose possible SALT observations of the fields of our selected targets. The $3 \sigma$ uncertainty of a Fermi LAT source is approximately 0.06 degrees. In such a region away from the Galactic plane, there are several hundreds of sources. A variable among them will likely be the counterpart. By carefully planning, approximately $2 / 3$ of our targets will be visible to SALT. Because of the goal of finding relatively bright variables, exposures will be from a few seconds to a few minutes in one band, which can be easily taken within the altitude restrictions of SALT. One set of observations of a target field can consist of short-exposure imaging over 2-3 nights, for the purpose of finding variables of several hours for compact binaries and one-day for wideorbit binaries. Once a candidate is found, targeted imaging and spectroscopy can be carried out to determine a binary's orbital period and properties for binary identification. In order to determine an orbital period by building up sufficient time sampling, we may take several exposures of a candidate in one night and repeat such observations over a few days or even several months because of the limited visibility windows of SALT. Such orbital information, once obtained, will greatly help searching for pulsation signals at radio or even $\gamma$-ray frequencies (e.g., [14, 15]). Based on the spectra from our Fermi LAT data analysis, all our selected targets are likely MSPs and thus far most Fermi MSPs are in binaries. The chance of identifying them through optical imaging is thus high. We have currently tested to obtain the data for several targets of the northern hemisphere, and soon we shall be able to learn the possible success rate of finding them from our test observing run.

\section{References}

[1] A. A. Abdo, M. Ajello, A. Allafort, L. Baldini, J. Ballet, G. Barbiellini, M. G. BAring, D. Bastieri, A. Belfiore, R. Bellazzini, And et Al., The Second Fermi Large Area Telescope Catalog of Gamma-Ray Pulsars , ApJS, 208 (2013), p. 17.

[2] F. Acero, M. Ackermann, M. Ajello, W. B. Albert, A. A nd Atwood, M. Axelsson, L. Baldini, G. Ballet, J. an d Barbiellini, D. Bastieri, and et Al., Fermi Large Area Telescope Third Source Catalog, ApJS, 218 (2015), p. 23.

[3] M. Ackermann, M. Ajello, W. B. Atwood, L. Baldini, J. Ballet, G. BarbiEllini, D. Bastieri, J. Becerra GonZalez, R. Bellazzini, E. Bissaldi, And 
ET AL., The Third Catalog of Active Galactic Nuclei Detected by the Fermi Large Area Telescope, ApJ, 810 (2015), p. 14.

[4] A. M. Archibald, I. H. Stairs, S. M. Ransom, V. M. Kaspi, V. I. Kondratiev, D. R. Lorimer, M. A. McLaughlin, J. Boyles, J. W. T. Hessels, R. Lynch, J. van Leeuwen, M. S. E. Roberts, F. Jenet, D. J. Champion, R. Rosen, B. N. Barlow, B. H. Dunlap, And R. A. Remillard, A Radio Pulsar/X-ray Binary Link, Science, 324 (2009), pp. 1411.

[5] W. B. Atwood, A. A. Abdo, M. Ackermann, W. Althouse , B. Anderson, M. Axelsson, L. Baldini, J. Ballet, D. L. Band, G. Barbiellini, and et Al., The Large Area Telescope on the Fermi Gamma-Ray Space Telescope Mission, ApJ, 697 (2009), pp. 1071-1102.

[6] C. G. Bassa, A. Patruno, J. W. T. Hessels, E. F. Keane, B. Monard, E. K. Mahony, S. Bogdanov, S. Corbel, P. G. Edwards, A. M. Archibald, G. H. Janssen, B. W. STAPPERS, AND S. TENDUlKAR, A state change in the low-mass X-ray binary XSS J12270-4859, MNRAS, 441 (2014), pp. 1825-1830.

[7] O. G. Benvenuto, M. A. De Vito, And J. E. Horvath, Understanding the Evolution of Close Binary Systems with Radio Pulsars, ApJL, 786 (2014), p. L7.

[8] H.-L. Chen, X. Chen, T. M. Tauris, and Z. Han, Formation of Black Widows and Redbacks-Two Distinct Populations of Eclipsing Binary Millisecond Pulsars, ApJ, 775 (2013), p. 27.

[9] K. S. Cheng, C. Ho, ANd M. Ruderman, Energetic radiation from rapidly spinning pulsars. I - Outer magnetosphere gaps. II - VELA and Crab, ApJ, 300 (1986), pp. 500-539.

[10] A. S. Fruchter, D. R. Stinebring, and J. H. Taylor, A millisecond pulsar in an eclipsing binary, Nature, 333 (1988), pp. 237-239.

[11] A. B. Hill, A. Szostek, S. Corbel, F. Camilo, R. H. D. Corbet, R. Dubois, G. Dubus, P. G. Edwards, E. C. Ferrara, M. Kerr, E. Koerding, D. Koziet, and Ł. STAWARZ, The bright unidentified $\gamma$-ray source 1FGL J1227.9-4852: can it be associated with a low-mass X-ray binary?, MNRAS, 415 (2011), pp. 235-243.

[12] A. G. Muslimov And A. K. Harding, High-Altitude Particle Acceleration and Radiation in Pulsar Slot Gaps, ApJ, 606 (2004), pp. 1143-1153.

[13] A. Patruno And A. L. Watts, Accreting Millisecond X-Ray Pulsars, ArXiv eprints:1206.2727, (2012).

[14] H. J. Pletsch, L. Guillemot, H. Fehrmann, B. Allen, M. Kramer, C. Aulbert, M. Ackermann, M. Ajello, A. De Angelis, W. B. Atwood, And et Al., Binary Millisecond Pulsar Discovery via Gamma-Ray Pulsations, Science, 338 (2012), pp. 1314-. 
[15] P. S. Ray, S. M. Ransom, C. C. Cheung, M. Giroletti, I. Cognard, F. Camilo, B. Bhattacharyya, J. Roy, R. W. Romani, And et Al., Radio Detection of the FermiLAT Blind Search Millisecond Pulsar J1311-3430, ApJL, 763 (2013), p. L13.

[16] M. T. Reynolds, P. J. Callanan, A. S. Fruchter, M. A. P. Torres, M. E. Beer, AND R. A. GIBBOns, The light curve of the companion to PSR B1957+20, MNRAS, 379 (2007), pp. 1117-1122.

[17] M. S. E. RoberTs, Surrounded by spiders! New black widows and redbacks in the Galactic field, in IAU Symposium, J. van Leeuwen, ed., vol. 291 of IAU Symposium, Mar. 2013, pp. 127-132.

[18] R. W. RomAni, 2FGL J1311.7-3429 Joins the Black Widow Club, ApJL, 754 (2012), p. L25.

[19] B. W. Stappers, A. M. Archibald, J. W. T. Hessels, C. G. Bassa, S. Bogdanov, G. H. Janssen, V. M. Kaspi, A. G. Lyne, A. Patruno, S. Tendulkar, A. B. Hill, And T. Glanzman, A State Change in the Missing Link Binary Pulsar System PSR J1023+0038, ApJ, 790 (2014), p. 39.

[20] B. W. Stappers, M. Bailes, A. G. Lyne, R. N. Manchester, N. D’Amico, T. M. TAuris, D. R. Lorimer, S. Johnston, And J. S. SAndhu, Probing the Eclipse Region of a Binary Millisecond Pulsar, ApJL, 465 (1996), p. L119.

[21] B. W. Stappers, M. H. VAN Kerkwijk, J. F. Bell, AND S. R. Kulkarni, Intrinsic and Reprocessed Optical Emission from the Companion to PSR J2051-0827, ApJL, 548 (2001), pp. L183-L186.

[22] J. Strader, L. Chomiuk, E. Sonbas, K. Sokolovsky, D. J. Sand, A. S. Moskvitin, And C. C. Cheung, 1FGL J0523.5-2529: A New Probable Gamma-Ray Pulsar Binary, ApJL, 788 (2014), p. L27.

[23] P. H. T. Tam, C. Y. Hui, R. H. H. Huang, A. K. H. Kong, J. Takata, L. C. C. Lin, Y. J. YANG, K. S. CHENG, AND R. E. TAAM, Evidence for Gamma-ray Emission from the Low-mass X-ray Binary System First J102347.6+003841, ApJL, 724 (2010), pp. L207-L211.

[24] D. J. Thompson, Gamma ray astrophysics: the EGRET results, Reports on Progress in Physics, 71 (2008), p. 116901.

[25] J. R. Thorstensen And E. Armstrong, Is FIRST J102347.6+003841 Really a Cataclysmic Binary?, AJ, 130 (2005), pp. 759-766.

[26] Z. Wang, A. Tziamtzis, D. L. Kaplan, and D. Chakrabarty, Optical Modulation in the X-Ray Binary $4 U$ 1543-624 Revisited, ArXiv e-prints, (2014).

[27] K. E. Williamson, S. G. Jorstad, A. P. Marscher, V. M. Larionov, P. S. Smith, I. Agudo, A. A. Arkharov, D. A. Blinov, C. Casadio, N. V. Efimova, and et Al., Comprehensive Monitoring of Gamma-Ray Bright Blazars. I. Statistical Study of Optical, X-Ray, and Gamma-Ray Spectral Slopes, ApJ, 789 (2014), p. 135. 
[28] Y. XING AND Z. WANG, Fermi/Observation of the Transitional Pulsar Binary XSS J122704859, ApJ, 808 (2015), p. 17. 